\title{
Análisis preliminar de las relaciones entre el nivel de condición física y el apoyo parental percibido para la práctica deportiva en adolescentes con sobrepeso y obesidad Preliminary analysis of the relationship between physical fitness level and perceived parental support for sports practice in overweight and obese adolescents \\ *Sebastián López-Serrano, **Nuno Eduardo Marques de Loureiro, *Sara Suarez-Manzano, *Manuel Jesús de la Torre- Cruz \\ *Universidad de Jaén (España), **Instituto Politécnico de Beja (Portugal)
}

Resumen. El objetivo de este estudio residió en conocer si el apoyo percibido por los progenitores para la práctica de actividad física se relacionaba con el sexo y con el nivel de condición física de que presentaba un grupo de adolescentes con sobrepeso y obesidad. Un total de 177 adolescentes pertenecientes a instituciones escolares públicas, con edades comprendidas entre los 12 y los 16 años de edad, con índice de masa corporal promedio de 29.03 participaron en este estudio. Los resultados de este estudio revelaron que los mayores niveles de apoyo general parental hacia la práctica deportiva se observaron en los chicos y chicas que presentaban una elevada capacidad aeróbica. Asimismo, las chicas atribuyeron a sus progenitores un mayor grado de apoyo guiado con relación a sus compañeros varones. Con los resultados arrojados por este estudio podemos concluir pues, que existe una relación positiva entre condición física y apoyo parental percibido.

Palabras clave: sobrepeso, obesidad, apoyo parental, condición física.

\begin{abstract}
The objective of this study was to assess if parents' perceived support towards the practice of physical activity was related to gender and to physical fitness level in a group of overweight and obese adolescents. A total of 177 adolescents enrolled in public school institutions, aged between 12 and 16 years old, with an average body mass index of 29.03, participated in this study. The results of this study revealed that the highest levels of general parental support for sports were observed in boys and girls with high aerobic capacity. Likewise, girls attributed their parents a greater degree of guided support compared to their male peers. With the results of this study, we can conclude that there is a positive relationship between physical fitness and perceived parental support.
\end{abstract}

Key words: overweight, obesity, parental support, fitness.

\section{Introducción}

El incremento en el porcentaje de niños y adolescentes que muestran un exceso de peso corporal se ha convertido en un asunto que ha captado el interés y ha alertado a los sistemas educativos y de salud pública de los países desarrollados (Wang \& Lobstein, 2006). La relación que mantiene el sobrepeso y la obesidad infanto-juvenil con una serie de problemas físicos (cardiovasculares, respiratorios, ortopédicos) y psicológicos (ansiedad, depresión, baja autoestima) ha sido puesta de manifiesto en diferentes estudios (Buttitta, Iliescu, Rousseau \& Guerrien, 2014; Sanders, Han \& Baker, 2015; Rodríguez-Ayllon et al., 2018). Además del peso corporal en sí mismo, la menor frecuencia de práctica de actividad física, así como el peor nivel de condición física exhibido por los chicos y chicas con sobrepeso y obesidad respecto a sus compañeros normopesos, contribuyen al mantenimiento de estos problemas (Pahkala et al., 2013; Ruedl, Franz, Frühauf, Kopp, Niedermeier, Drenowatz, \& Greier, 2018).

Aunque parece existir consenso en las consecuencias negativas a nivel físico y psicológico vinculadas al exceso de peso corporal y la ausencia de práctica de actividad física, mayor cantidad de debate ha suscitado la discusión relativa a si el sobrepeso y la obesidad infato-juvenil es la causa o la consecuencia de la inactividad física y la consiguiente pobre condición física asociada a la inactividad (Metcalf, Hosking, Jeffery, Voss, Henley, \& Wilkin, 2011; Pahkala et al., 2013).

Fecha recepción: 18-03-19. Fecha de aceptación: 26-07-19 Sara Suarez-Manzano ssm00016@gmail.com
Diferentes autores plantean que el exceso de peso podría actuar como causa dado que, los chicos y chicas que presentan una estructura corporal más voluminosa, tendrían mayores dificultades para lograr el dominio de las habilidades motrices implicadas en la práctica deportiva lo que, a su vez, convertiría estas actividades en poco atractivas (Metcalf et al., 2011; Pojskic \& Eslami, 2018), propiciando un menor disfrute con su realización y el progresivo abandono de su práctica, completando un círculo vicioso que conduciría al incremento de peso corporal.

No obstante, la realización de actividad física no solo se encuentra asociada al peso corporal. Otros factores de naturaleza personal (autoeficacia, competencia percibida, autoconcepto físico) y contextual-social (disponibilidad de lugares de práctica, el apoyo de los iguales o la influencia de madres y padres) han mostrado ser correlatos de su práctica (Babic, Morgan, Plotnikoff, Londsdale, White \& Lubans, 2014; Lindwall, Asci \& Crocker, 2014; Rhee, Jelalian, Boutelle, Dickstein, Seifer \& Wing, 2016; Yao \& Rhodes, 2015). Entre los segundos, la familia ha sido considerada como una fuente de socialización y apoyo para que niños y adolescentes sean físicamente activos. Entre las distintas formas en las que madres, padres y hermanos promueven la realización de actividad física se encuentran las muestras de ánimo (verbal y no verbal), la co-participación, la facilitación (la provisión de transporte y pago de cuotas) y el modelado (Gustafson \& Rhodes, 2006; Yao \& Rhodes, 2015). En términos generales, los resultados obtenidos en diferentes estudios revelan que el apoyo parental, en sus distintas modalidades, se relaciona de forma positiva con la práctica de actividad física de los hijos (Cheng, Mendonça \& de Farias-Júnior, 2014; Morrissey, Wenthe, Letuchy, Levy \& Janz, 2012; Wright, Wilson, Griffin \& Evans, 2010). 
Si bien el apoyo parental para la práctica de actividad física ha sido evaluado a través de la información aportada tanto por los progenitores (apoyo ofrecido) como por los hijos (apoyo percibido), las opiniones de unos y otros suelen ser discordantes (Brunet et al., 2014). Al respecto, investigaciones recientes concluyen que la atribución realizada por los hijos es un mejor predictor de la frecuencia de actividad física que el juicio ofrecido por los adultos (BarrAnderson, Robinson-O’Brien, Haines, Hannan \& NeumarkSztainer, 2010; Haines, Neumark-Sztainer, Hannan \& Robinson-O’Brien, 2008).

Adicionalmente, algunos estudios han planteado si la cantidad de apoyo difiere entre los jóvenes que presentan sobrepeso y peso normal. Al respecto, Zabinski, Saelens, Stein, Hayden-Wade \& Wilfley (2003) y De Bourdeaudhuij Lefevre, Deforche, Wijndaele, Matton \& Philippaerts (2005) obtuvieron que los chicos y chicas con sobrepeso atribuyeron a sus progenitores menores niveles de apoyo para la práctica de actividad física con relación a sus iguales normopesos. Sin embargo, poco se sabe de hasta qué punto estas muestras de apoyo percibido pueden diferir en función del nivel de condición física mostrado por adolescentes con sobrepeso y obesidad. Así pues, el objetivo del presente estudio residió en conocer si existían diferencias significativas en la cantidad de apoyo parental para la práctica de actividad física que un grupo de adolescentes con sobrepeso y obesidad atribuía a sus progenitores en función de su nivel de potencia aeróbica. Adicionalmente, se examinaron las posibles diferencias en función del sexo del participante. Se hipotetizó que los chicos y chicas cuyos niveles de capacidad aeróbica fuesen más elevados informarían de mayores muestras de apoyo recibido por parte de sus progenitores.

\section{Materialy método}

\section{Participantes}

Un total de 388 adolescentes que presentaban sobrepeso u obesidad, de acuerdo con su sexo y edad cronológica, formaron parte de este estudio. De este conjunto inicial se obtuvieron datos completos para un total de 177 jóvenes (45.6\%), procedentes de ocho centros de Educación Secundaria Obligatoria, que conformaron la muestra final del estudio (53.1\% chicos). La edad promedio de los participantes fue de 14.20 años $(D T=1.27)$, dentro de un rango comprendido entre los 12 y 16. La estatura y peso corporal promedio alcanzaron los valores de 1.65 metros $(D T=.09)$ y 79.50 kilogramos $(D T=11.30)$, respectivamente. El valor medio del índice de masa corporal de los participantes empleando la fórmula propuesta por Quetelet, [IMC = peso $(\mathrm{kg}) /$ altura $\left(\mathrm{m}^{2}\right)$ ] ascendió a 29.03 (Cole \& Lobstein, 2012).

\section{Instrumentos}

Medidas antropométricas.

La estatura y peso se registraron con un tallímetro portátil SECA 214 Ltd Germany® y una báscula ASIMED tipo B. Los datos se obtuvieron con los participantes descalzos y vestidos con ropa ligera.

\section{Capacidad aeróbica}

Prueba de ida y vuelta de 20 metros (Leger, Mercier, Gadoury \& Lambert, 1988). La tarea exige recorrer la distancia de 20 metros que separa dos líneas. La velocidad de carrera se controla mediante una señal acústica que se presenta a intervalos regulares, señal que se acelera con el transcurso del tiempo. De inicio, la señal se presenta con una frecuencia que equivale a 8.5 kilómetros/hora, frecuencia que se incrementa en 0.5 kilómetros/hora tras cada minuto de tiempo (un minuto equivale a un palier). La prueba concluye cuando el participante se detiene debido a la fatiga, o bien, cuando no consigue alcanzar por segunda vez consecutiva una de las líneas con la señal de audio. La puntuación registrada equivale al último medio palier completado.

Para determinar el nivel de potencia aeróbica del participante se calculó de inicio el valor de la mediana en el número de paliers completados en la prueba de ida y vuelta de 20 metros, en función del sexo (chica y chico) y edad de los participantes (de 12 a 16 años). De acuerdo con estos criterios los valores resultantes en el número de paliers para cada una de las edades fueron los siguientes: 12 años (2.75 vs 3.00), 13 años (2.50 vs 3.50), 14 años (2.50 vs 3.50), 15 años (3.00 vs 4.50) y 16 años ( 2.50 vs 5.50 ), respectivamente. Tomando como referentes estos valores, un participante era incluido en el grupo de buena capacidad aeróbica si el valor alcanzado, según su edad y sexo, era superior al arrojado por la mediana. Por el contrario, si este valor resultaba ser inferior se asignaba al grupo de pobre capacidad. Para comprobar la pertinencia de esta clasificación se realizó una prueba $t$ de medidas independientes que contrastó el número medio de paliers completados en función de los grupos establecidos. El resultado arrojó diferencias estadísticamente significativas, $t(171)=19.79, p<.001$, favorables a los jóvenes con mayor resistencia aeróbica $(M=4.98$ vs $M=2.36)$.

Escala de influencia parental sobre la actividad física

Se empleó una versión adaptada al castellano de la escala elaborada por Jago, Fox, Page, Brockman \& Thompson (2009). Está compuesta por 14 ítems que se agrupan en cuatro factores: apoyo parental general («Los adultos con los que vivo me trasladan hasta los lugares en los que practico actividad física»), actividad física parental presente ( «Los adultos con los que vivo realizan mucha actividad física a lo largo de la semana»), actividad física parental pasada («Los adultos con los que vivo solían realizar mucha actividad física a lo largo de la semana, pero, ahora no lo hacen») y apoyo dirigido («Los adultos con los que vivo me animan o instan a que sea físicamente activo/a»). Los participantes han de indicar a lo largo de una escala tipo Likert de cuatro puntos el grado de acuerdo ( 1 = totalmente en desacuerdo, 2 = bastante en desacuerdo, 3 = bastante de acuerdo y 4 = totalmente de acuerdo) mostrado con la afirmación. Se calcula una puntuación promedio por cada una de las dimensiones, correspondiendo un valor más elevado con una mayor percepción de influencia parental para la práctica de actividad física. La fiabilidad, entendida como consistencia interna, calculada mediante el estadístico á de Cronbach osciló entre los valores de .80 y .87 para las dimensiones actividad física parental pasada y presente, respectivamente. 


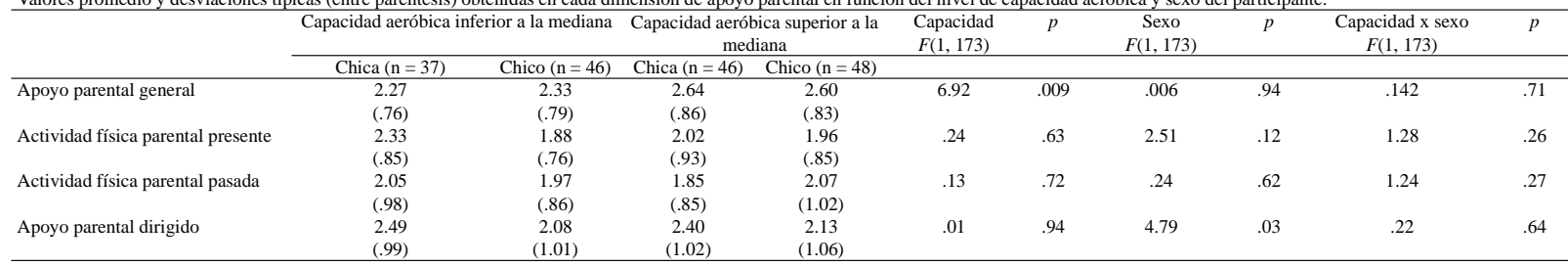

\section{Diseño y Procedimiento}

Este estudio, de naturaleza transversal, era parte de un proyecto de investigación más amplio destinado a conocer la existencia de las posibles relaciones entre el apoyo parental, la frecuencia de práctica de actividad física, la condición física y el bienestar psíquico en población adolescente. Inicialmente, se contactó con una veintena de centros de Educación Secundaria Obligatoria a quienes se explicó el objetivo general de la investigación. De todos ellos ocho accedieron a participar. El tipo de muestreo empleado fue por conveniencia, no probabilístico ni aleatorio. El equipo directivo de los centros participantes, padres y alumnos recibieron información más detallada de la investigación y mediante consentimiento informado aceptaron colaborar en este estudio. El protocolo fue aprobado por el Comité de Bioética de la Universidad de Jaén (referencia ABR.17/2). Los datos personales de los chicos y chicas participantes se reemplazaron por códigos alfanuméricos para garantizar el anonimato. Las medidas de antropometría, potencia aeróbica y autoinformes se aplicaron durante las diferentes clases de Educación Física bajo la supervisión de un miembro del equipo investigador. El estudio se adecua a la normativa española de investigación en humanos (Real Decreto 1090/2015 sobre ensayos clínicos), la legislación relativa a la protección de datos (Ley Orgánica 15/1999) y las directrices consensuadas en la Declaración de Helsinki (2013).

\section{Análisis estadístico}

Los análisis estadísticos se realizaron mediante el programa estadístico SPSS v. 21 para Windows. Los resultados se presentan como medias y desviaciones típicas. Las pruebas utilizadas fueron á de Cronbach para el análisis de la consistencia interna (fiabilidad) de los ítems incluidos en cada factor, la prueba de Levene para el examen de la igualdad de las varianzas error y el análisis de varianza de dos factores.

\section{Resultados}

Se realizaron cuatro análisis de varianza 2x2 empleando como variables independientes el sexo del participante (chico vs chica) y la capacidad aeróbica (baja vs alta). Las variables dependientes fueron las puntuaciones promedio obtenidas en cada una de las dimensiones que conforman la escala de influencia parental hacia la actividad física. En todos y cada uno de los análisis conducidos la prueba de Levene que examina la igualdad de las varianzas error no arrojó la existencia de diferencias estadísticamente significativas, $F(3,173)=1.00, p=.39$, para la de mayor valor en la medida dependiente actividad física pasada.

El análisis estadístico conducido para la variable depen- diente apoyo parental general arrojó la existencia de un efecto principal estadísticamente significativo de la variable independiente capacidad aeróbica, $F(1,173)=6.92, p=.009$, $c^{2}$ $=.04$. De esta forma, los chicos y chicas incluidos en el grupo que mostró un mejor rendimiento en la prueba de ida y vuelta de 20 metros percibían en sus progenitores mayores índices de apoyo para la práctica de actividad física $(M=2.62$ vs $M$ $=2.30)$. Ni la variable sexo, $F(1,173)=.01, p>.05$, ni la interacción capacidad aeróbica x sexo, $F(1,173)=.14, p>.05$, resultaron estadísticamente significativas.

Atendiendo a la actividad física parental presente y pasada, ni el efecto principal de la variable independiente capacidad aeróbica, $F(1,173)=.24, p>.05$ y $F(1,173)=.13, p>.05$, respectivamente, ni la variable independiente sexo, $F(1,173)$ $=2.51, p>.05$ y $F(1,173)=.24, p>.05$, ni la interacción capacidad aeróbica $x$ sexo, $F(1,173)=1.28, p>.05$ y $F(1,173)$ $=1.24, p>.05$, resultaron ser estadísticamente significativas.

Por último, en relación a la variable dependiente apoyo dirigido se observó un efecto principal estadísticamente significativo de la variable sexo, $F(1,173)=4.79, p=.03, c^{2}=.03$. En este sentido, las chicas expresaron recibir una guía más estructurada por parte de sus madres y padres para la práctica de actividad física ( $M=2.45$ vs $M=2.11)$. Ni el efecto principal de la variable capacidad aeróbica, $F(1,173)=.01, p$ $>$.05, ni la interacción capacidad aeróbica x sexo, $F(1,173)=$ $.21, p>.05$, alcanzaron la significación estadística. En la tabla número 1 se muestran los valores promedio y desviaciones típicas.

\section{Discusión}

Este estudio tuvo por objetivo examinar la posible relación existente entre el nivel de potencia aeróbica de un grupo de adolescentes con sobrepeso y obesidad y el grado de apoyo parental que afirmaban recibir de sus padres para la práctica de actividad física. Asimismo, se examinó si esta relación difería en función del sexo del participante. Los resultados obtenidos revelaron que los chicos y chicas que presentaban una mayor resistencia aeróbica, evaluada a través de la prueba de la prueba de ida y vuelta de 20 metros, atribuían a sus progenitores un mayor grado de apoyo parental general. Este resultado está en sintonía con el obtenido recientemente por De la Torre-Cruz, Ruiz-Ariza, SuárezManzano \& Martínez-López (2018) en cuyo estudio se constató que el índice de masa corporal del adolescente moderaba la relación entre la capacidad aeróbica y apoyo parental instrumental que los padres informaban ofrecer a sus hijos para la práctica de actividad física. De acuerdo con De la Torre-Cruz et al. (2018), la relación entre capacidad aeróbica y apoyo parental instrumental fue positiva, pero, de manera particular cuando el índice de masa corporal de los adoles- 
centes era inferior al promedio de los participantes. En una línea similar, Nock, Levers-Landis, Dajani, Knight, Rigda, Narasimhan \& Uli (2016), examinaron las relaciones existentes entre diferencias dimensiones del clima familiar (cohesión, conflicto y expresividad emocional), la autoeficacia percibida y la condición física en una muestra de adolescentes con sobrepeso y obesidad. Los resultados obtenidos revelaron la existencia de una relación negativa y estadísticamente significativa entre el nivel de cohesión y expresividad emocional y el tiempo de recuperación de la tasa cardíaca base tras una prueba de esfuerzo máximo de tres minutos de duración que servía como indicador de un mayor nivel de condición física.

Los resultados obtenidos confirman parcialmente lo hipotetizado dado que, de las cuatro formas evaluadas, solo una de ellas, el apoyo parental general se asocia con la potencia aeróbica de los participantes. No obstante, este hallazgo parece estar en sintonía con el estudio de Yao \& Rhodes (2015) sobre los correlatos parentales de la práctica de actividad física de niños y adolescentes. Tras la realización de un meta-análisis con más de un centenar de investigaciones los autores concluyeron que, mientras que el efecto del modelado fue pequeño, el del apoyo hacia la práctica de actividad física, particularmente el aliento y estímulo parental, resultó ser moderado.

Adicionalmente, se observó la existencia de un efecto principal estadísticamente significativo del sexo del participante sobre la dimensión apoyo guiado. En este sentido, las chicas percibían que sus progenitores les instaban a ser físicamente más activas en comparación con sus compañeros varones. Este resultado no se alinea con el obtenido por Zabinski et al. (2003) en cuyo estudio las chicas con sobrepeso y obesidad percibían un menor grado de apoyo parental para la práctica de actividad física con relación a sus iguales normopesas, diferencias que no se apreciaron en el caso de sus compañeros varones. Aunque este mayor apoyo percibido podría considerarse positivo dado que las chicas adolescentes con exceso de peso corporal han sido consideradas como un grupo de riesgo, este resultado debe interpretarse con cautela. Al respecto, Davison \& Deane (2010) concluyeron que las chicas que afirmaban sentirse más animadas por sus progenitores para ser físicamente activas con el propósito de reducir peso expresaban un menor grado de diversión con la realización de actividad física, así como una mayor preocupación por su imagen corporal. En una línea similar, Heitzler, Martin, Duke \& Huhman (2006) afirmaron que una mayor exigencia o demanda parental tenía un efecto más pernicioso que estimulante para la práctica de actividad física de sus hijas adolescentes, dado que tales demandas generaban mayores índices de ansiedad en las jóvenes que, en muchas ocasiones, se traducía en el abandono de la actividad.

Explorar las posibles relaciones entre la resistencia aeróbica junto a otros indicadores de condición física y el apoyo parental percibido para la práctica de actividad física en jóvenes con sobrepeso y obesidad es de indudable interés. En este sentido, la mejor condición física exhibida por los adolescentes, tal vez asociada a una actividad física más frecuente dadas las relaciones positivas existentes entre ambas, se vincula con una atribución más elevada de apoyo parental que, a su vez, puede propiciar la implicación futura de una mayor cantidad de actividad física. Este razonamiento parece congruente con los resultados recientes obtenidos por Zarychta, Mullan \& Luszcynska (2016). En su estudio, un grupo de adolescentes con sobrepeso y obesidad que atribuyó a sus padres una mayor adhesión a ciertas conductas saludables (mantener una dieta y practicar actividad física), incrementó durante el transcurso de un año estos mismos comportamientos lo que se tradujo en una disminución del índice de masa corporal trece meses después. De esta forma, una alimentación sana, la práctica de actividad física y la adopción de un estilo de vida más saludable mediaron de forma significativa la relación entre los comportamientos saludables percibidos en los progenitores y el índice de masa corporal de adolescentes con sobrepeso y obesidad.

Es necesario destacar algunas fortalezas y limitaciones de este estudio. Entre las primeras cabe citar las características de la muestra de estudio y su considerable tamaño (adolescentes con sobrepeso y obesidad), así como la evaluación objetiva de la capacidad aeróbica de los participantes mediante la prueba de ida y vuelta de 20 metros. Respecto a las limitaciones, la naturaleza transversal del estudio no permite establecer relaciones de causa y efecto entre el nivel de potencia aeróbica mostrado por chicas y chicos y el apoyo parental percibido para la práctica de actividad física. Además, los problemas inherentes a las medidas de autoinforme pueden considerarse como otra debilidad.

\section{Conclusiones}

La potencia aeróbica de un grupo de adolescentes con sobrepeso y obesidad se relaciona de forma positiva con el apoyo general que afirman recibir de sus padres para la práctica de actividad física, pero, no así el modelado parental, tanto presente como pasado, atribuido. Asimismo, las chicas afirman sentirse más dirigidas o guiadas en este apoyo en comparación con sus compañeros varones. Dada la naturaleza preliminar de este estudio se precisa de un mayor número de investigaciones que nos permitan conocer otras variables asociadas al apoyo parental hacia la práctica de actividad física, ofrecido y percibido, en niños y adolescentes con exceso de peso corporal.

\section{Agradecimientos}

La realización de este trabajo ha sido posible gracias a la subvención obtenida por el proyecto «Actividad física en adolescentes y contexto familiar. Estudio transversal y programa de intervención en padres e hijos de dos años de duración» financiado por el Plan de Ayuda a la I+D+I (2016) de la Universidad de Jaén (código 2016/00095). Además, se recibió apoyo del Programa de Formación de Profesorado Universitario, implementado por el Ministerio de Educación, Cultura y Deporte del Gobierno de España (grant number AP-2016-07226). Finalmente, los autores desean agradecer la participación desinteresada de los estudiantes, madres y padres, así como docentes para la materialización de este estudio. 


\section{Referencias}

Babic, M. J., Morgan, P. J., Plotnikoff, R. C., Lonsdale, C., White, R. L., \& Lubans, D. R. (2014). Physical activity and physical self-concept in youth: Systematic review and meta-analysis. Sports Medicine, 44, 1589-1601. doi:10.1007/s40279-0140229-z

Barr-Anderson, D. J., Robinson-O’Brien, R., Haines, J., Hannan, P., \& Neumark-Sztainer, D. (2010). Parental report versus child perception of familial support: which is more associated with child physical activity and television use? Journal of Physical Activity and Health, 7(3), pp. 364-368. doi: 10.1123/ jpah.7.3.364

Brunet, J. Sabiston, C. M., O’Loughlin, J., Mathieu, M., Tremblay, A., Barnett, T. A., \& Lambert, M. (2014). Perceived parental social support and moderate-to-vigorous physical activity in children at risk of obesity. Research Quarterly for Exercise and Sport, 85, pp. 198-207. doi: 10.1080/ 02701367.2014.893049

Buttitta, M., Iliescu, C., Rousseau, A., \& Guerrien, A. (2014). Quality of live in overweight and obese children and adolescents: a literature review. Quality of Life Research, 23(4), 1117-1139. doi:10.1007/s11136-013-0568-5

Cole, T. J., \& Lobstein, T. (2012). Extended international (IOFT) body mass index cut-offs for thinnes, overweight and obesity. Pediatric Obesity, 7, 284-294.

Cheng, L. A., Mendonça, G., \& de Farias Júnior, J. C. (2014). Physical activity in adolescents: analysis of the social influence of parents and friends. Jornal de Pediatria, 90, pp. 35-41. Doi: 10.1016/j.jped.2013.05.006

Davison, K. K., \& Deane, G. D. (2010). The consequence of encouraging girls to be active for weight loss. Social Science \& Medicine, 70(4), pp. 518-525. doi: 10.1016/ j.socscimed.2009.10.061

De Bourdeaudhuij, I., Lefevre, J., Deforche, B., Wijndaele, K., Matton, L., \& Philippaerts, R. (2005). Physical activity and psychosocial correlates in normal weight and overweight 11 to 19 year olds. Obesity Research, 13, pp. 1097-1105. doi: 10.1038/oby.2005.128

De la Torre-Cruz, M. J., Ruiz-Ariza, A., Suárez-Manzano, S., \& Martínez-López, E. J. (2018). Does body mass index moderate the relationship between parental support, fitness, and physical activity practice in adolescents? Psicología Conductual/ Behavioral Psychology, 26(1), pp. 95-113.

Gustafson, S. L., \& Rhodes, R. E. (2006). Parental correlates of physical activity in children and early adolescents. Sports Medicine, 36(1), pp. 79-97. doi: 10.2165/00007256-20063601000006

Haines, J., Neumark-Sztainer, D., Hannan, P., \& Robinson-O’Brien, R. (2008). Child versus parent report of parental influences on children's weight-related attitudes and behaviors. Journal of Pediatric Psychology, 33(7), pp. 783-788. doi: 10.1093/jpepsy/ jsn016

Heitzler, C. D., Martin, S. L., Duke, J., \& Huhman, M. (2006) Correlates of physical activity in a national sample of children aged 9-13 years. Preventive Medicine, 42, 254-260. doi: 10.1016/j.ypmed.2006.01.010

Jago, J., Fox, K. R., Page, A. S., Brockman, R., \& Thompson, J. L. (2009). Development of scales to assess children's perceptions of friends and parental influences on physical activity. International Journal of Behavioral Nutrition and Physical Activity, 6(67), pp. 1-10. doi:10.1186/1479-5868-6-67

Leger, L. A., Mercier, D., Gadoury, C., \& Lambert, J. (1988). The multistage 20 metre shuttle run test for aerobic fitness. Journal of Sports Sciences, 6(2), pp. 93-101. doi: 10.1080/ 02640418808729800

Lindwall. M,, Asci, H., \& Crocker, P. R. E. (2014). The physical self in motion: Within-person change and associations of change in self-esteem, physical self-concept, and physical activity in adolescent girls. Journal of Sport and Exercise Psychology, 36, 551-563. doi:10.1123/jsep.2013-0258

Metcalf, B. S., Hosking, J., Jeffery, A. N., Voss, L. D., Henley, W., \& Wilkin, T. J. (2011). Fatness leads to inactivity, but inactivity does not lead to fatness: a longitudinal study in children (EarlyBird 45). Archives of Disease in Childhood, 96(10), 942947. doi: 10.1136/adc.2009.175927

Morrissey, J. L., Wenthe, P. J., Letuchy, E. M., Levy, S. M., \& Janz, K. F. (2012). Specific types of family support and adolescent non-school physical activity levels. Pediatric Exercise Science, 24, pp. 333-346. doi: 10.1123/pes.24.3.333

Nock, N. L., Levers-Landis, C. E., Dajani, R., Knight, D., Rigda, A., Narasimhan, S., \& Uli, N. (2016). Physical activity selfefficacy and fitness: Family environment relationship correlates and self-esteem as a mediator among adolescents who are overweight or obese. Childhood Obesity, 12(5), 360-367. doi:10.1089/chi2016.0007

Pahkala, K., Hernelahti, M., Heinonen, O. J., Raittinen, P., Hakanen, M., Lagström, H., Viikari, J. S., ... \& Simell, O. (2013). Body mass index, fitness and physical activity from childhood through adolescence. British Journal of Sports Medicine, 47(2), 71-77. doi:10.1136/ bjsports-2011-090704

Pojskic, H., \& Eslami, B. (2018). Relationship between obesity, physical activity, and cardiorespiratory fitness levels in children and adolescents in Bosnia and Herzegovina: An analysis of gender differences. Frontiers in Physiology, 9:1734. doi:10.3389/fphys.2018.01734

Rhee, K. E., Jelalian, E., Boutelle, K., Dickstein, S., Seifer, R., \& Wing, R. (2016). Warm parenting associated with decreasing or stable child BMI during treatment. Childhood Obesity, 12(2), 94-102. doi:10.1089/chi.2015.0127

Rodriguez-Ayllon, M., Cadenas-Sanchez, C., Esteban-Cornejo, I., Migueles, J. H. Mora-Gonzalez, J., Henriksson, P., MartínMatillas,..., \& Ortega, F. B. (2018). Physical fitness and psychological health in overweigth/obese children: A crosssectional study from the ActiveBrains projects. Journal of Science and Medicine in Sport, 21, 179-184. doi: 10.1016/ j.jsams.2017.09.019

Ruedl, G., Franz, D., Frühauf, A., Kopp, M., Niedermeier, M., Drenowatz, C., \& Greier, K. (2018). Development of physical fitness in Austrian primary school children: A longitudinal study among overweight and non-overweight children over 2.5 years. Wiener Klinische Wochenschrift, 130, 321-327. doi: 10.1007/ s00508-018-1336-x

Sanders, R. H., Han, A., Baker, J. S., \& Cobley, S (2015). Childhood obesity and its physical and psychological co-morbidities: a systematic review of Australian children and adolescents. European Journal of Pediatrics, 174(6), 715-746. doi: 10.1007/ s00431-015-2551-3

Wang, Y. \& Lobstein, T. (2006). Worldwide trends in childhood overweight and obesity. International Journal of Pediatric Obesity, 1(1), 11-25. doi: 10.1080/17477160600586747

Wright, M. S., Wilson, D. K., Griffin, S., \& Evans, A. (2010). A qualitative study of parental modeling and social support for physical activity in underserved adolescents. Health Education Research, 25, pp. 224-232. doi: 10.1093/her/cyn043

Yao, C. A., \& Rhodes, R. E. (2015). Parental correlates in child and adolescent physical

activity: a meta-analysis. International Journal of Behavioral Nutrition and Physical Activity, 12, pp. 1-38. doi: 10.1186/ s12966-015-0163-y

Zabinski, M. F., Saelens, B. E., Stein, R. I., Hayden-Wade, H. A., \& Wilfley, D. E. (2003). Overweight children's barriers to and support for physical activity. Obesity Research, 11, 238-246. doi: 10.1038/oby.2003.37

Zarychta, K., Mullan, B., \& Luszczynska, A. (2016). It doesn't matter what they say, it matters how they behave: Parental influences and changes in body mass among overweight and obese adolescents. Appetite, 96, 47-55. 